\title{
Sensitivity Analysis for the Encaged Turbine Concept in Oscillating Water Column Plants
}

\author{
Demos P. Georgiou, Kypros F. Milidonis, and Eleutherios N. Georgiou \\ Thermal Engines Laboratory, Department of Mechanical Engineering and Aeronautics, Polytechnic School, University of Patras, \\ 26500 Patras, Achaia, Greece \\ Correspondence should be addressed to Kypros F. Milidonis, milidon@mech.upatras.gr
}

Received 31 October 2012; Accepted 2 December 2012

Academic Editors: H. Ramos and S. Senthilarasu

Copyright () 2012 Demos P. Georgiou et al. This is an open access article distributed under the Creative Commons Attribution License, which permits unrestricted use, distribution, and reproduction in any medium, provided the original work is properly cited.

\begin{abstract}
Oscillating water column plants are one of the most popular wave energy device types. Prototype OWC units have been operating in various parts of the world since the mid-1980s and such developers have more field experience of this technology than any other relevant plant. The most common turbine used is the self-rectifying Well's turbine which has a rather low peak efficiency if compared to other designs but was preferred in terms of its simplicity and cycle performance. The present study exploits the merits of a new concept for the power extraction process, that of an encaged turbine for OWC plants, which allows conventional highefficiency turbines to be employed in such plants. This is achieved by guiding the pressurized air into a sequence of three chambers, creating a unidirectional closed air circuit through the turbine. A theoretical model is deployed simulating the operation of the plant and a sensitivity analysis is carried out for the design and working parameters. Results indicate that the power extraction efficiency may exceed the $50 \%$ level in a real plant.
\end{abstract}

\section{Introduction}

The exploitation of the energy contained in the earth's oceans has been proposed several times in the past, especially the energy that is carried in the ocean's waves. Several different wave energy devices for harvesting the wave energy have been proposed [1,2], but the main concept under investigation are the oscillating water columns. The principle of operation of the traditional oscillating water column is rather simple, extracting useful mechanical energy by operating on the U-manometer principle, where water in one of the "legs" oscillates under the action of the waves, while that in the second acts as a piston on the air cavity above. In a real plant, a hollow reinforced concrete structure-pneumatic chamber-communicates with the sea (and the incident waves) by a submerged opening in its front wall $[3,4]$. The incident waves act on the water column contained in the lower part of the capture chamber, which in turn causes alternate air flow to and from a duct that contains the turbine which drives the electric generator.
Almost all prototype plants of OWC employ the Well's turbine as the power delivering system, which has the advantage of not requiring rectifying valves because of its ability to rotate unidirectional despite the air flow direction. Though results in terms of efficiency so far have been rather disappointing by the usage of Well's turbine, mainly due to hysteretic characteristics of the symmetrically blades in a reciprocating flow [5]. The turbine's symmetrical configuration with respect to the plane perpendicular to the rotor axis, in order to operate in a bidirectional reciprocating flow has the inherent disadvantages of relatively low efficiency and poor starting characteristics. During the nearly zero flow conditions imposed by the flow reversal the turbine rotation absorbs power in order to overcome the viscous drag. Low or even negative torque occurs at relatively small flow rates and in addition, at high flow rates the symmetric airfoils lead to an early blade stall at rather low incidence angles. On the other hand, a number of other air turbines with different configurations have been patented and improved over the last years [6-8]. However, their superiority against the Well's 


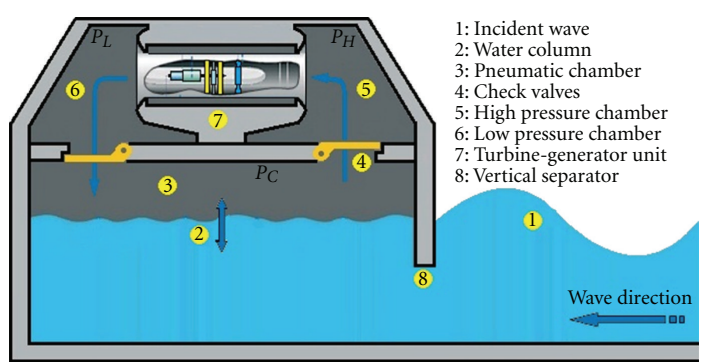

FIgURE 1: The encaged turbine concept in OWC.

turbine has not yet been proved. In addition, Setoguchi and Takao [9] made an extensive review concerning the current status of self-rectifying air turbines for wave energy conversion.

The present study examines further the characteristics of a different type of oscillating water column plant, that of a fully encaged turbine concept. The concept for such a plant was first introduced by Georgiou et al. [10]. This early investigation studied the influence of the flow discharge coefficient through the turbine and determined the plant's efficiency in respect to this coefficient. The distinctive characteristic of the fully encaged structure drives the pressurized air to a loop through a sequence of three chambers, so that the flow passing through the power turbine maintains a constant direction. This is implemented through the action of non-return valves that regulate the mass fluxes among the three chambers and ensures that the back-forth oscillation of the air through the turbine is avoided. This implies that the power turbine will face a continuous unidirectional flow, thus avoiding the hysteresis problems associated with the Well's concept. In addition, the fully encaged concept and the resulting closed air circuit will eliminate the noise generated by the exhausting air with the auditory and visual disturbances for the surrounding environment. The current study describes the operation of the plant and performs a sensitivity analysis concerning the main characteristics and design parameters in order to obtain initial estimations about dimensioning, proper functioning, and wave energy absorption efficiency.

\section{The Encaged Oscillating Water Column Turbine Concept}

The concept of the encaged turbine consists of a hollow structure which is divided into three separate chambers, the main (3) and two supporting ones (5) and (6) as illustrated in Figure 1. It must be noted that none of the three chambers communicates with external environment, and the system functions only with the air trapped inside. The main chamber (3) communicates with the sea and the incident waves by a submerged vertical separator (8) in its front wall. Incident waves (1) act on the water column (2) contained in the pneumatic chamber where the oscillatory motion of the water causes a difference in air pressure with respect to the outside atmosphere. As the level of the water column rises, air is pressurized inside the pneumatic chamber and

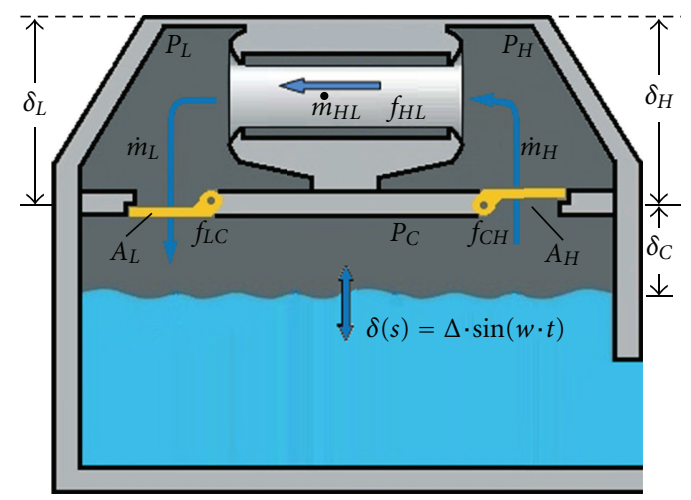

Figure 2: Characteristics and design parameters of the idealized concept.

forced to pass to the high pressure chamber (5) through as set of check non-return valves (4) that separates the two chambers. These valves are self-operated by the pressure acting on their surface and regulate the mass fluxes among the chambers. The pressure chamber air is then expanded to the second supporting chamber, called low pressure chamber (6), through a duct connecting the two chambers. Inside the duct a turbine-generator unit (7) is located. As the wave retracts, the water column level decreases and air is forced to pass from the low pressure chamber to the main one through a second set of non-return valves. The sequence is repeated with the frequency of the sea waves, so that the flow through the turbine maintains the same directional path and drives the air turbine in a unidirectional mode. Operation of the system as it is described ensures that the flow through the turbine maintains constant direction and the trapped air is forced to circulate in the sequence of the three chambers.

\section{The Theoretical Model}

Although the air inside the chambers is compressed, the presence of such a large water surface and the significant foaming-droplet formation associated with its oscillation most likely will result into a nearly isothermal process rather than an isentropic. This has been shown to be true for conventional OWC plants and the present concept does not anticipate much different operating conditions. In a strict formulation the isothermal process could be enforced through water spraying into the three chambers.

In any case, the present analysis makes a "worst scenario" hypothesis and assumes that the compressions are practically isothermal. A mathematical model of the operation of the proposed plant has been developed. The model for the proposed plant is illustrated in Figure 2 where the main characteristics and working parameters are illustrated.

Assuming that the vertical water column oscillates in a sinusoidal form with a frequency $w$ and amplitude $\Delta$ so that

$$
\delta_{s}=\Delta \cdot \sin w t
$$


The mass conservation law for each of the three cavities leads to:

$$
\begin{aligned}
\frac{d m_{H}}{d t} & =\dot{m}_{H}-\dot{m}_{H L}, \\
\frac{d m_{L}}{d t} & =\dot{m}_{H L}-\dot{m}_{L}, \\
\frac{d m_{C}}{d t} & =\dot{m}_{L}-\dot{m}_{H}
\end{aligned}
$$

To simplify the problem, the analysis will be carried out in terms of nondimensional parameters. These parameters are derived by dividing the main dimensional quantities (i.e., chamber masses $m_{i}$ ) by the corresponding initial values, that is,

$$
M_{i}=\frac{m_{i}}{m_{i 0}}
$$

where index " 0 " indicates the initial (calm sea) state and index " $i$ " indicates relevant chamber, that is,

$i=H$ for high pressure chamber,

$i=L$ for low pressure chamber, and

$i=C$ for the pneumatic chamber as illustrated in Figure 2.

But

$$
m_{0}=\rho_{0} \cdot V_{0}=\frac{P_{0} \cdot V_{0}}{R \cdot T_{0}} .
$$

Hence the nondimensional forms take the forms shown in (6). The corresponding nondimensional form of the time parameter is given in (7):

$$
\begin{gathered}
M_{C}=\frac{m_{C}}{m_{C 0}}=\frac{m_{C}}{P_{0} \cdot V_{0}} R T_{0}=\frac{m_{C}}{A_{C} \cdot \delta_{C}} \frac{R T_{0}}{P_{0}}, \\
M_{L}=\frac{m_{L}}{A_{L} \cdot \delta_{L}} \frac{R T_{0}}{P_{0}}, \\
M_{H}=\frac{m_{H}}{A_{H} \cdot \delta_{H}} \frac{R T_{0}}{P_{0}}, \\
\tau=w \cdot t .
\end{gathered}
$$

By applying the nondimensional parameters to (2) we get

$$
\begin{gathered}
\left(m_{H 0} \cdot w\right) \frac{d M_{H}}{d \tau}=\left(m_{H 0} \cdot w\right)\left(\dot{M}_{H}-\dot{M}_{H L}\right) \\
\Longrightarrow \frac{d M_{H}}{d \tau}=\left(\dot{M}_{H}-\dot{M}_{H L}\right) .
\end{gathered}
$$

Similarly working, the rest nondimensional equations become

$$
\begin{gathered}
\frac{d M_{L}}{d \tau}=\left(\frac{m_{H 0}}{m_{L 0}}\right)\left(\dot{M}_{H L}-\dot{M}_{L}\right), \\
\frac{d M_{C}}{d \tau}=\left(\frac{m_{L 0}}{m_{C 0}}\right) \dot{M}_{L}-\left(\frac{m_{H 0}}{m_{C 0}}\right) \dot{M}_{H} .
\end{gathered}
$$

The parameter $\dot{m}_{H}$ represents the mass flux from chamber $C$ to chamber $H, \rho$ the air density inside each chamber, $P_{C}$ and $P_{H}$ the pressures in chambers $C$ and $H$, respectively, while $f_{C H}$ introduces the discharge coefficient. The flow discharge is evaluated by employing the following relationship:

$$
\begin{gathered}
\dot{m}_{H}=\rho_{C} \cdot A_{H} \sqrt{\frac{2\left(P_{C}-P_{H}\right)}{\rho_{C}}} \cdot f_{C H} \quad\left(\text { if } P_{H}<P_{C}\right), \\
\dot{m}_{H}=0 \quad\left(\text { if } P_{H} \geq P_{C}\right) .
\end{gathered}
$$

Similarly the mass flux $\dot{m}_{L}$ between the chambers $L$ and $C$ is calculated by

$$
\begin{gathered}
\dot{m}_{L}=\rho_{L} \cdot A_{L} \sqrt{\frac{2\left(P_{L}-P_{C}\right)}{\rho_{L}}} \cdot f_{C L} \quad\left(\text { if } P_{L}>P_{C}\right), \\
\dot{m}_{L}=0 \quad\left(\text { if } P_{L} \leq P_{C}\right),
\end{gathered}
$$

where

$$
m_{L}=\frac{P_{L} \cdot V_{L}}{R \cdot T_{L}}, \quad \rho_{L}=\frac{m_{L}}{V_{L}} .
$$

Cross-sectional areas $A_{H}$ and $A_{L}$ correspond to the area of the non-return valves that allows the air to travel between chambers. In practice these planes will be composed out of boards capable of turning inside only one chamber and supported on transverse beams. Hence, nearly the entire plane will be a "non-return valve". In the present analysis they are taken to be equal to the cross-section of the corresponding rectangular chambers. The discharge coefficients $f_{C L}$ and $f_{C H}$ were taken to be equal to a constant value of $f_{C L}=f_{C H} \simeq 0.9$. The mass flux from chamber $H$ to $L\left(\dot{m}_{H L}\right)$ (i.e., through the power generating turbine) is calculated as follows:

$$
\begin{gathered}
\dot{m}_{H L}=\rho_{H} \cdot A_{H L} \sqrt{\frac{2\left(P_{H}-P_{L}\right)}{\rho_{H}}} \cdot f_{H L} \quad\left(\text { if } P_{H} \geq P_{L}\right), \\
\dot{m}_{H L}=-\rho_{L} \cdot A_{H L} \sqrt{\frac{2\left(P_{L}-P_{H}\right)}{\rho_{L}}} \cdot f_{H L} \quad\left(\text { if } P_{L}>P_{H}\right) .
\end{gathered}
$$

The area $A_{H L}$ represents the open cross-sectional area between the high pressure and low pressure chamber and $f_{H L}$ indicates the coefficient of discharge among the two chambers. This coefficient in a real plant will be deduced from the load to flow coefficient relationship. Now by applying the nondimensional parameters to (10) and solving for $\dot{M}_{H}$ with $P_{H}<P_{C}$ we have

$$
\begin{gathered}
m_{H 0} \cdot w \cdot \dot{M}_{H}=f_{C H} \cdot A_{H} \sqrt{2 \cdot \rho_{C 0} \rho_{C}^{*} P_{0}\left(P_{C}^{*}-P_{H}^{*}\right)}, \\
w \cdot \dot{M}_{H}=\left(\frac{f_{C H} \cdot A_{H} \sqrt{2 \cdot \rho_{C 0} \cdot P_{0}}}{m_{H 0}}\right) \cdot \sqrt{\rho_{C}^{*}\left(P_{C}^{*}-P_{H}^{*}\right)}, \\
w \cdot \dot{M}_{H}=\frac{f_{C H} \cdot A_{H} \sqrt{2 \cdot\left(P_{0} / R \cdot T_{0}\right) \cdot P_{0}}}{P_{0} \cdot V_{H 0} / R \cdot T_{0}} \cdot \sqrt{\rho_{C}^{*}\left(P_{C}^{*}-P_{H}^{*}\right)}, \\
w \cdot \dot{M}_{H}=\sqrt{2} \cdot f_{C H} \cdot\left(\frac{A_{H}}{V_{H 0}}\right) \sqrt{R \cdot T_{0}} \cdot \sqrt{\rho_{C}^{*}\left(P_{C}^{*}-P_{H}^{*}\right)},
\end{gathered}
$$


where

$$
\rho_{C}^{*}=\frac{\rho_{C}}{\rho_{C 0}}, \quad P_{C}^{*}-P_{H}^{*}=\frac{P_{C}-P_{H}}{P_{0}} .
$$

And finally by using the fact that $V_{H 0}=V_{H}=\delta_{H} \cdot A_{H}$ (assuming a rectangular shape for the chambers) we get

$$
\dot{M}_{H}=\sqrt{2} \cdot f_{C H} \cdot\left(\frac{\sqrt{R \cdot T_{0}}}{w \cdot \delta_{H}}\right) \cdot \sqrt{\rho_{C}^{*}\left(P_{C}^{*}-P_{H}^{*}\right)}
$$

for $P_{C}^{*}>P_{H}^{*}$ else $\dot{M}_{H}=0$.

Similarly, from (12) for $P_{L}>P_{C}$ and $V_{L 0}=\delta_{L} \cdot A_{L}$ we get

$$
\dot{M}_{L}=\sqrt{2} \cdot f_{L C} \cdot\left(\frac{\sqrt{R \cdot T_{0}}}{w \cdot \delta_{L}}\right) \cdot \sqrt{\rho_{L}^{*}\left(P_{L}^{*}-P_{C}^{*}\right)}
$$

for $P_{L}^{*}>P_{C}^{*}$ else $\dot{M}_{L}=0$.

For $\dot{M}_{H L}$ using (15) for $P_{H}>P_{L}$ we have

$$
\begin{gathered}
m_{H 0} \cdot w \cdot \dot{M}_{H L}=f_{H L} \cdot A_{H L} \sqrt{2 \cdot \rho_{H 0} \rho_{H}^{*} P_{0}\left(P_{H}^{*}-P_{C}^{*}\right)}, \\
\dot{M}_{H L}=f_{H L} \cdot \sqrt{2} \cdot \frac{A_{H L}}{m_{H 0} \cdot w} \cdot \sqrt{\rho_{H 0} \cdot P_{0}} \cdot \sqrt{\rho_{C}^{*}\left(P_{C}^{*}-P_{H}^{*}\right)},
\end{gathered}
$$

$$
\begin{array}{r}
\dot{M}_{H L}=f_{H L} \cdot \sqrt{2} \cdot\left(\frac{\sqrt{R \cdot T_{0}}}{w \cdot \delta_{H}}\right) \cdot \sqrt{\rho_{H}^{*}\left(P_{H}^{*}-P_{L}^{*}\right),} \\
\text { for } P_{H}^{*}>P_{L}^{*}, \\
\dot{M}_{H L}=f_{H L} \cdot \sqrt{2} \cdot\left(\frac{\sqrt{R \cdot T_{0}}}{w \cdot \delta_{L}}\right) \cdot \sqrt{\rho_{L}^{*}\left(P_{L}^{*}-P_{H}^{*}\right)}, \\
\text { if } P_{L}^{*}>P_{H}^{*} .
\end{array}
$$

The nondimensional pressures $P_{C}^{*}, P_{H}^{*}, P_{L}^{*}$ for each cavity can be obtained by dividing the dimensional $P_{C}, P_{H}, P_{L}$ quantities by the corresponding initial values; that is, for the pneumatic chamber we have

$$
P_{C}^{*}=\frac{P_{C}}{P_{C 0}}=\frac{m_{C}}{m_{C 0}} \frac{R}{R} \frac{T_{C}}{T_{C 0}} \frac{V_{C 0}}{V_{C}} .
$$

By introducing the nondimensional temperature quantity $\Theta_{C}$, and since $V_{C 0}=V_{C}$, then the $P_{C}^{*}$ finally becomes

$$
\Longrightarrow P_{C}^{*}=M_{C}\left(\Theta_{C}+\left(1-\Theta_{C}\right) \frac{T_{R}}{T_{C 0}}\right) \frac{\delta_{C}}{\delta_{C}-\Delta \cdot \sin \tau} .
$$

Here $T_{R}$ refers to a temperature reference value and was set to $T_{R}=273,15 \mathrm{~K}$. For the high pressure chamber:

$$
\begin{aligned}
P_{H}^{*} & =\frac{P_{H}}{P_{0}}=\frac{m_{H}}{m_{H 0}} \frac{R}{R} \frac{T_{H}}{T_{H 0}} \frac{V_{H 0}}{V_{H}}, \\
& \Longrightarrow P_{H}^{*}=M_{H}\left(\Theta_{H}+\left(1-\Theta_{H}\right) \frac{T_{R}}{T_{0}}\right) .
\end{aligned}
$$

Similarly, by assuming a low pressure chamber, we get

$$
\Longrightarrow P_{L}^{*}=\frac{P_{L}}{P_{0}}=M_{L}\left(\Theta_{L}+\left(1-\Theta_{L}\right) \frac{T_{R}}{T_{0}}\right) .
$$

The nondimensional terms $\rho_{C}^{*}, \rho_{H}^{*}, \rho_{L}^{*}$ of the air density in each chamber can be obtained the same way:

$$
\begin{gathered}
\rho_{C}^{*}=\frac{\rho_{C}}{\rho_{C 0}}=\frac{m_{C}}{m_{C 0}} \frac{V_{C 0}}{V_{C}}=M_{C} \frac{1}{1-\left(\Delta / \delta_{C}\right) \sin \tau}, \\
\rho_{H}^{*}=\frac{\rho_{H}}{\rho_{H 0}}=\frac{m_{H}}{m_{H 0}}=M_{H}, \\
\rho_{L}^{*}=\frac{\rho_{L}}{\rho_{L 0}}=\frac{m_{L}}{m_{L 0}}=M_{L} .
\end{gathered}
$$

\section{The Sensitivity Analysis}

The analysis was implemented by solving the system of governing equations simultaneously. As it was mentioned before, the coefficients of discharge $f_{C L}$ and $f_{C H}$ were taken to be constant and equal to $f_{C L}=f_{C H} \simeq 0$,9. The initial value of the temperature in each chamber was set to be $T_{o}=290 \mathrm{~K}$, while the sensitivity analysis employed two plant sizes for the relevant calculations. The first size concerned a hydraulic model where $\delta_{H}=\delta_{L}=1 \mathrm{~m}$. For the second size, a realistic plant was assumed with $\delta_{H}=\delta_{L}=5 \mathrm{~m}$ and similar analogies for other dimensions. The calculations involved the concurrent integration of (8) and (9) and the mass flux relationships (19)-(22b). For this study, the free parameters under investigation are the oscillation frequency $w$ and the height $\delta_{c}$ of the air column above the oscillating water column. A nondimensional time step $(d \tau)$ equal to 0.001 was selected for the calculations. The influence of the coefficient of discharge $f_{H L}$ between the high and low pressure chamber was investigated by Georgiou et al. [10]. For the present study this was taken to be $f_{H L}=0.001$. This was deduced from a typical load-flow coefficient relationship of an axial turbine of a high (92\%) efficiency. The quotient $\Delta / \delta_{c}$ was set to be equal to 0.2 .

Figures 3 and 4 illustrate the comparison of the nondimensional pressures $P_{C}^{*}, P_{H}^{*}, P_{L}^{*}$ in each chamber for $w=2$ and $w=6$ (Figure 3 ) and $\delta_{C}=1 \mathrm{~m}$ and $\delta_{C}=5 \mathrm{~m}$ (Figure 4 ).

The angular frequency does not affect the pressure evolution inside the three chambers, but the relative chamber height does. The nature of this evolution is common to all cases. As the pressure in chamber $C$ increases, air starts to flow into the high pressure chamber $H$ and $P_{H}^{*}$ follows $P_{C}^{*}$ until it reaches its maximum value. At that point the wave retracts, so that both $P_{H}^{*}$ and $P_{C}^{*}$ start to decrease although $P_{C}^{*}$ drops faster than $P_{H}^{*}$. This happens because the air trapped in the high pressure chamber has to pass into the low pressure one and the wave retraction does not affect it since the valves communicating with the $C$ chamber are now closed. Simultaneously as $P_{H}^{*}$ increases, $P_{L}^{*}$ start to increase too because of the flow $\dot{m}_{H L}$ between the two chambers. In a specific point, $P_{L}^{*}$ pressure starts to follow the pressure loss form of the $P_{C}^{*}$ until it reaches to its minimum value.

The corresponding influence of these two parameters is illustrated in Figures 5 and 6 for the same set of free parameters. The analysis reveals that the nondimensional mass fluxes are not of a sinusoidal nature. This deviation tends to be greater as the angular frequency and the chamber size increases. The mass fluxes, $\dot{M}_{H L}, \dot{M}_{H}, \dot{M}_{L}$, are governed 


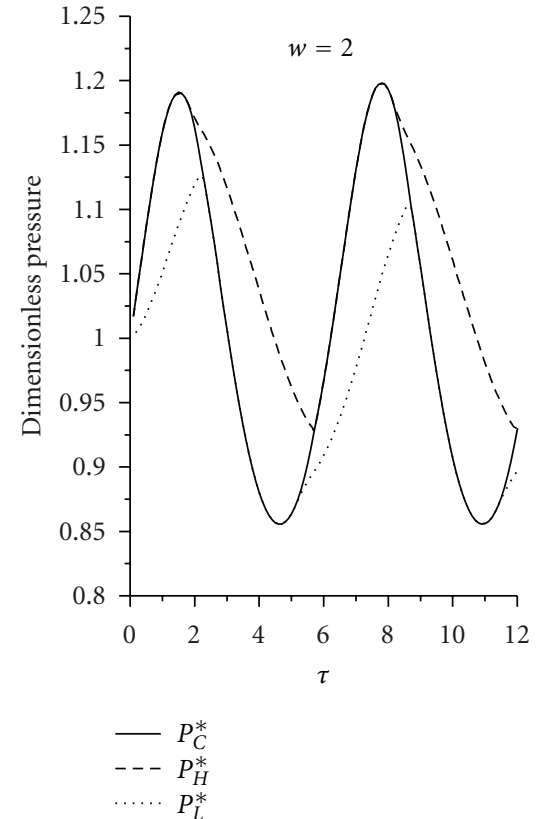

(a)

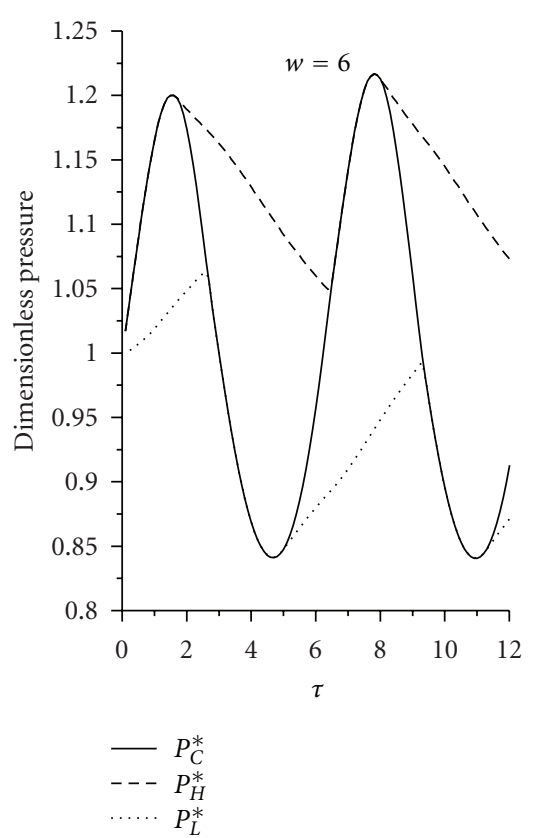

(b)

Figure 3: Pressures $P_{C}^{*}, P_{H}^{*}, P_{L}^{*}$ for different values of oscillation frequency $w$ as a function of dimensionless time $\tau$.

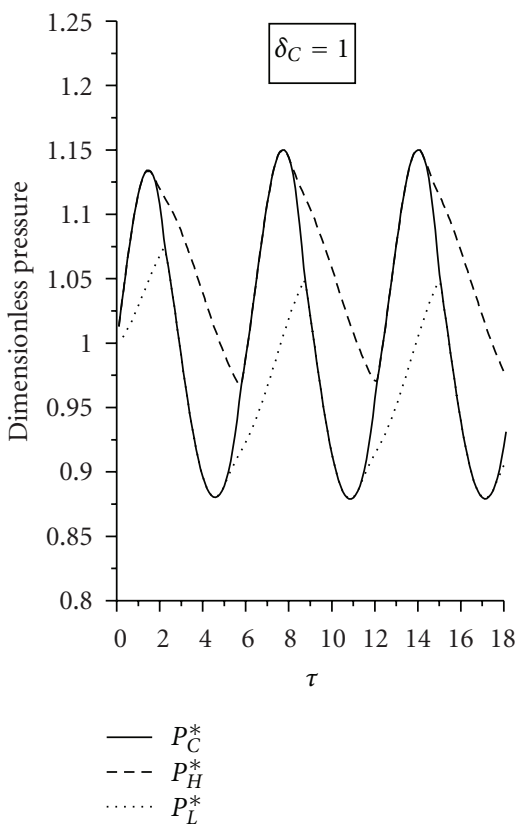

(a)

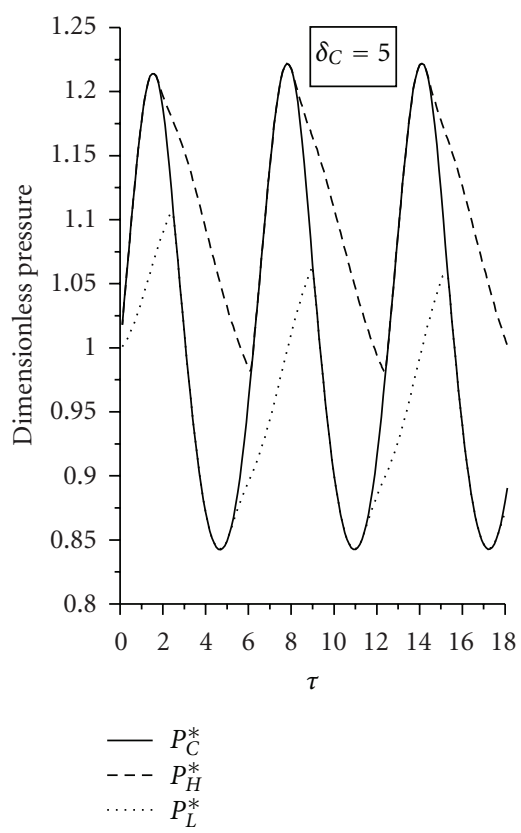

(b)

Figure 4: Pressures $P_{C}^{*}, P_{H}^{*}, P_{L}^{*}$ for different values of parameter $\delta_{c}$ as a function of dimensionless time $\tau$.

by the three equations (19), (20), and (22a) and (22b) where each of them is regulated by three different terms. The first term, that is, $\left(\sqrt{2} \cdot f_{C H}\right)$ in (19), is regulated by the opening characteristics of the valves, the second term, $\left(\sqrt{R \cdot T_{0}} / w \cdot \delta_{H}\right)$ by the "geometric" characteristics of the water column, while the third part, $\left(\sqrt{\rho_{C}^{*}\left(P_{C}^{*}-P_{H}^{*}\right)}\right)$, by the performance of the neighboring chambers. The same implies for the rest $\dot{M}_{H L}$ and $\dot{M}_{L}$. Thus, the oscillation frequency $w$ affects not only the mass fluxes but the dimensionless time $\tau$ as well. The parameter $\delta_{c}$ does not appear in any of (8) and (9) but it 


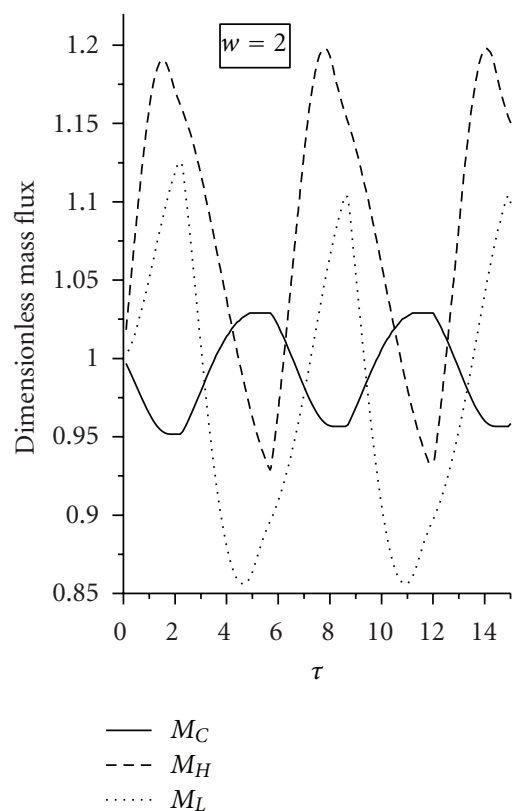

(a)

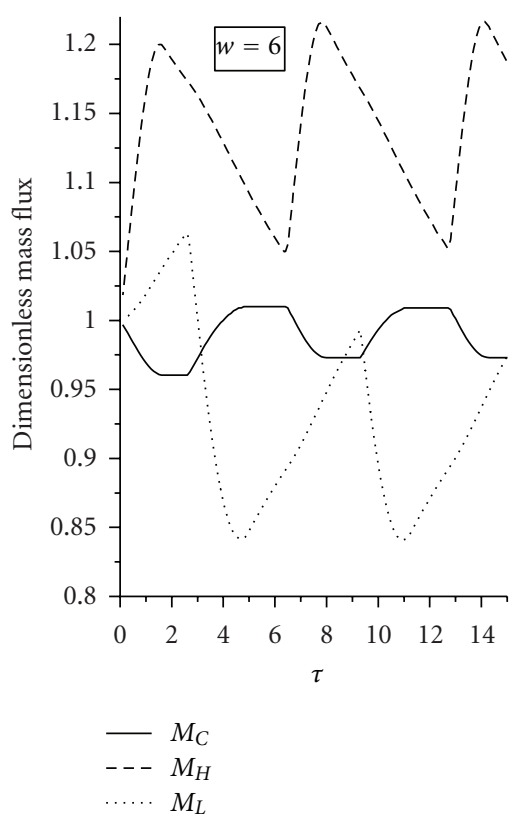

(b)

Figure 5: Dimensionless mass fluxes $\dot{M}_{C}, \dot{M}_{H}, \dot{M}_{L}$ for different values of the oscillation frequency $w$ as a function of the dimensionless time $\tau$.

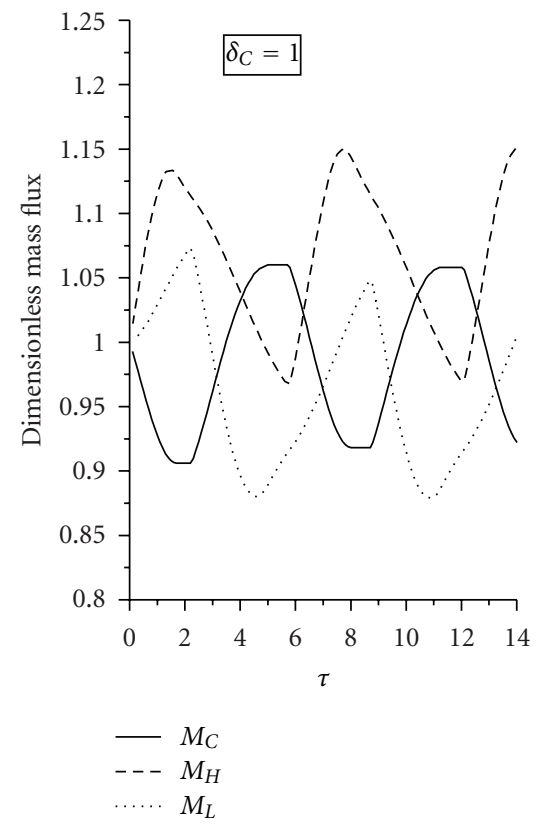

(a)

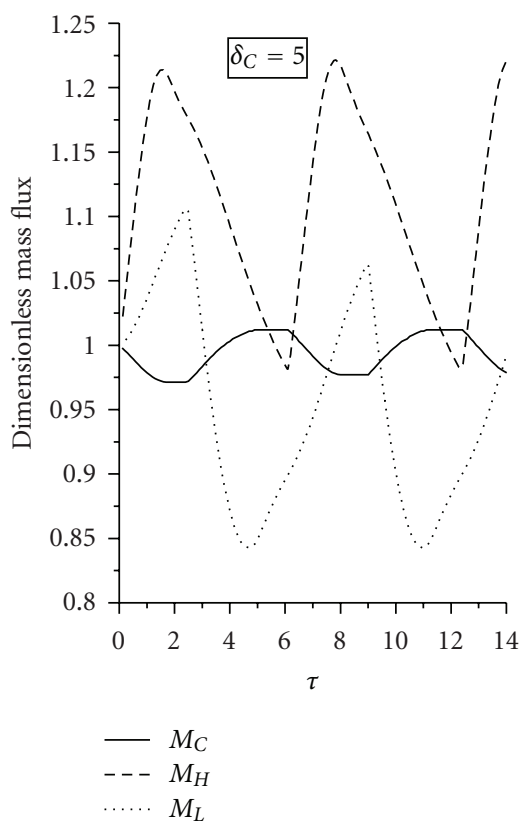

(b)

Figure 6: Dimensionless mass fluxes $\dot{M}_{C}, \dot{M}_{H}, \dot{M}_{L}$ for different values of the parameter $\delta_{c}$ as a function of the dimensionless time $\tau$.

does indirectly affect the $\dot{M}_{H L}, \dot{M}_{H}, \dot{M}_{L}$ values due to their dependence on the $\dot{M}_{C}$, in respect to (19), (20), (22a), and (22b).

The pressure difference $P_{H}^{*}-P_{L}^{*}$ as a function of the dimensionless time is illustrated in Figure 8 for different values of $w$ and $\delta_{c}$, while Figure 9 illustrates the nondimensional instant generation of useful power in the turbine.
The nondimensional instant turbine power was calculated by employing

$$
W_{\text {turb }}=\left(\frac{\dot{m}_{H L}}{w \cdot m_{c}}\right)\left(\frac{P_{H}-P_{L}}{P_{0}}\right) .
$$

The important thing to be noticed here is the fact that the nondimensional instant power $W_{\text {turb }}$ is always positive. 


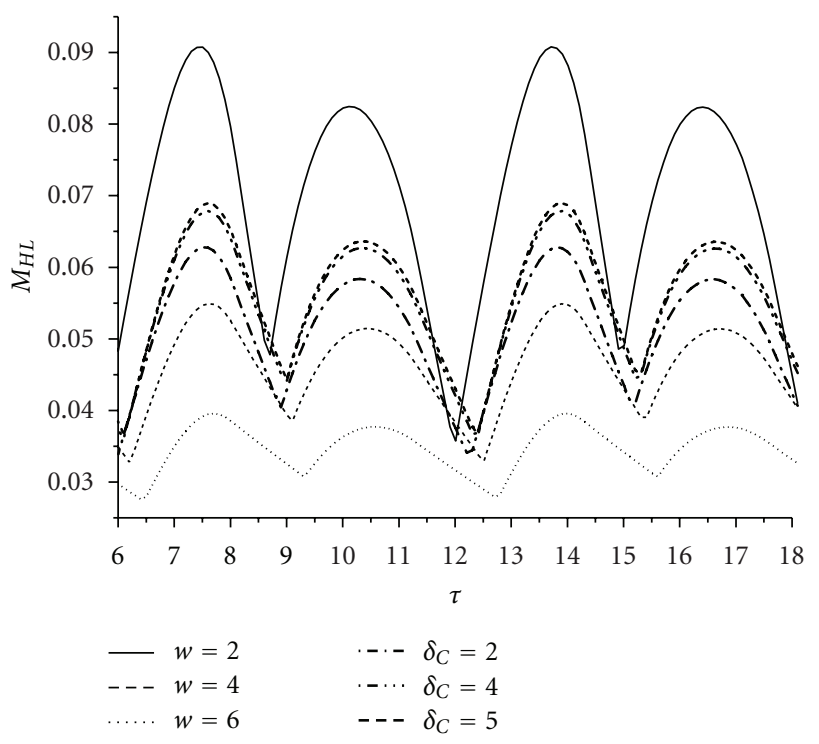

Figure 7: The dimensionless mass flux $\dot{M}_{H L}$ for different values of the parameter $\delta_{c}$ and the oscillation frequency $w$ as a function of the dimensionless time $\tau$.

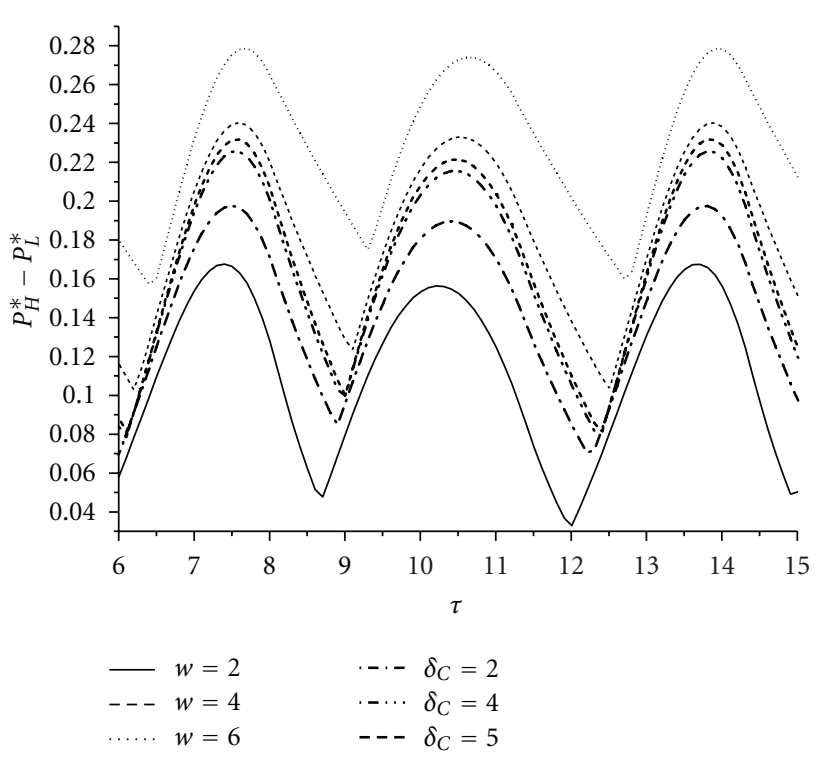

Figure 8: The pressure difference $P_{H}^{*}-P_{L}^{*}$ for different values of the parameter $\delta_{c}$ and oscillation frequency $w$ as a function of the dimensionless time $\tau$.

The same implies for the pressure difference between the high and low pressure chamber as it seems in Figure 8. The positive pressure difference verifies the one way air flux with no backflows. The nondimensional mass air flow $\dot{M}_{H L}$ shown in Figure 7 also supports the elimination of the back-forth oscillation of the air through the turbine since it takes only positive values.

The efficiency of the plant was obtained through the integration over a time period $\Delta \tau=2 \pi$. Figure 10 illustrates

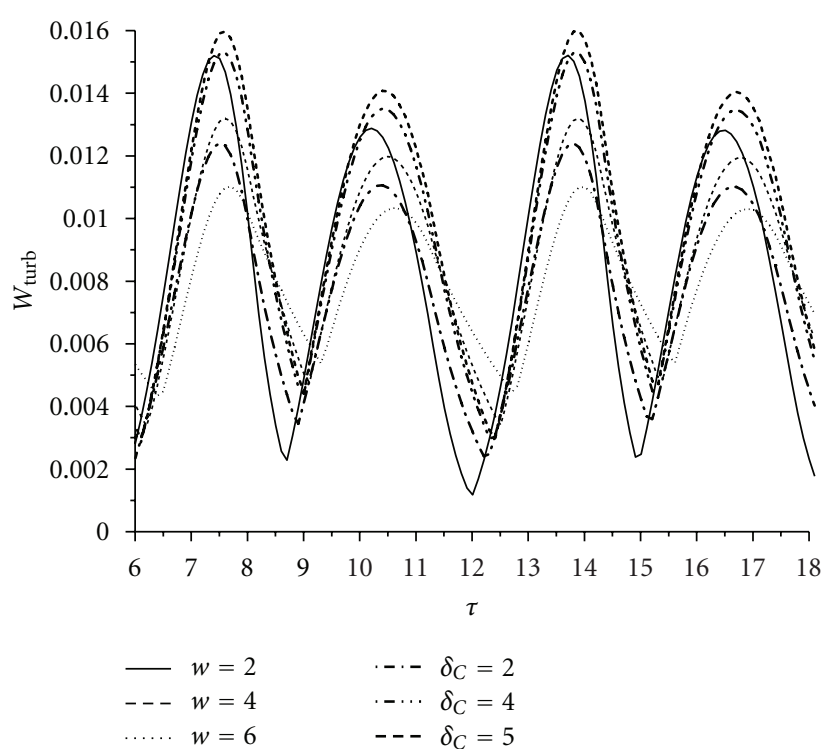

Figure 9: The dimensionless instant turbine power $W_{\text {turb }}$ for different values of the parameter $\delta_{c}$ and the oscillation frequency $w$ as a function of the dimensionless time $\tau$.

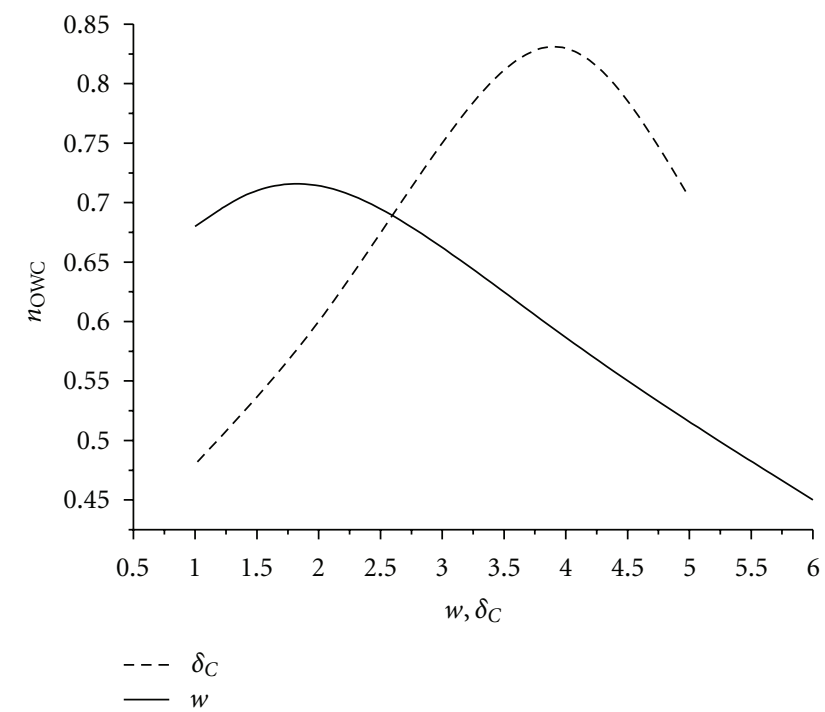

FIGURE 10: Influence of the parameter $\delta_{c}$ and oscillation frequency $w$ on the plant's efficiency.

the efficiency of the system as a function of the variables $\delta_{c}$ and $w$. The efficiency is defined as

$$
n_{\mathrm{owc}}=\frac{\int_{0}^{\Delta \tau} W d t}{\int_{0}^{\delta_{c}} P_{H} A_{C} d \delta_{c}} .
$$

As it can be seen, the proposed concept efficiency exhibits a similar performance compared with conventional OWC concepts where the ideal analysis efficiency may exceed $80 \%$ at resonance conditions. This condition is defined by the peaking efficiency point. In a well designed plant this resonance must coincide with the corresponding resonance 
between the impinging sea waves and the oscillating water inside the main chamber.

In addition to the above observations, it should be pointed out that the pressure deviation inside the chambers is of order 0.1 bars for the small unit and 0.2 bars for the larger. These levels have been observed in conventional OWC plants where relevant analyses and operating experience indicate that the isothermal process, perfect gas model predicts fairly accurately the actual operation of these plants.

\section{Conclusions}

A theoretical model is developed to simulate the encaged turbine concept for oscillating water column plants. The sensitivity analysis was carried out in order to investigate the influence of the oscillation frequency $w$ and the height $\delta_{c}$ of the air column above the oscillating water column, on the overall plant operation. The analysis reveals that both, the oscillation frequency $w$ and height $\delta_{c}$ are of great importance for proper dimensioning of the high and low pressure chamber and pneumatic chamber for a real plant application. In addition, the advantages over the conventional OWC plants in terms of efficiency have been shown. By avoiding the hysteresis effects on the power turbine the new concept promises an overall plant efficiency of the order of or above the $50 \%$ levels. The analysis showed that the elimination of air flow reversal through the turbine is possible and thus, high efficiency conventional turbines may be employed for the energy extraction process. In addition, due to the fully encaged concept, the system does not need to communicate with the external environment, minimizing thus the auditory and visual disturbances for the surrounding environment. As a result the proposed concept is preponderant because it can be "hidden" without changing the visual landscape from natural to industrial scenery.

\section{References}

[1] D. Ross, Power From the Waves, Oxford University Press, New York, NY, USA, 1995.

[2] A. Clément, P. McCullen, A. Falcão et al., "Wave energy in Europe: current status and perspectives," Renewable and Sustainable Energy Reviews, vol. 6, no. 5, pp. 405-431, 2002.

[3] A. Falcaõ, M. Sabino, T. Whittaker, and A. Lewis, "Design of a shoreline wave power pilot plant for the island of pico, azores," in Proceedings of the 2nd European Wave Power Conference, pp. 87-93, Lisbon, Portugal, 1995.

[4] T. Heath, T. Whittaker, and C. Boake, "The design, construction and operation of the limpet wave energy converter (Islay Scotland)," in Proceedings of 4th European Wave Energy Conference, pp. 49-55, Aalborg, Denmark, 2000.

[5] T. Setoguchia, Y. Kinouea, T. H. Kima, K. Kanekoa, and M. Inoueb, "Hysteretic characteristics of wells turbine for wave power conversion," Renewable Energy, vol. 28, no. 13, pp. 2113-2127, 2003.

[6] K. Kaneko, T. Setoguchi, and S. Raghunathan, "Self-rectifying turbines for wave energy conversion," International Journal of Offshore and Polar Engineering, vol. 2, no. 3, pp. 238-240, 1992.

[7] S. Anand, V. Jayashankar, S. Nagata, K. Toyota, M. Takao, and T. Setoguchi, "Turbines for wave energy plants," in Proceedings of the 8th International Symposium on Experimental and Computational Aerothermodynamics of Internal Flows, Lyon, France, 2007.

[8] B. Pereiras, F. Castro, A. E. Marjani, and M. A. Rodríguez, "An improved radial impulse turbine for OWC," Renewable Energy, vol. 36, no. 5, pp. 1477-1484, 2011.

[9] T. Setoguchi and M. Takao, "Current status of self rectifying air turbines for wave energy conversion," Energy Conversion and Management, vol. 47, no. 15-16, pp. 2382-2396, 2006.

[10] P. D. Georgiou, F. K. Milidonis, P. T. Xenos, and F. N. Theodoropoulos, "The encaged turbine concept in oscillating water column plants," in Proceedings of 3th International Conference of Renewable Energy Sources and Energy Efficiency, pp. 176-188, Nicosia, Cyprus, 2011. 

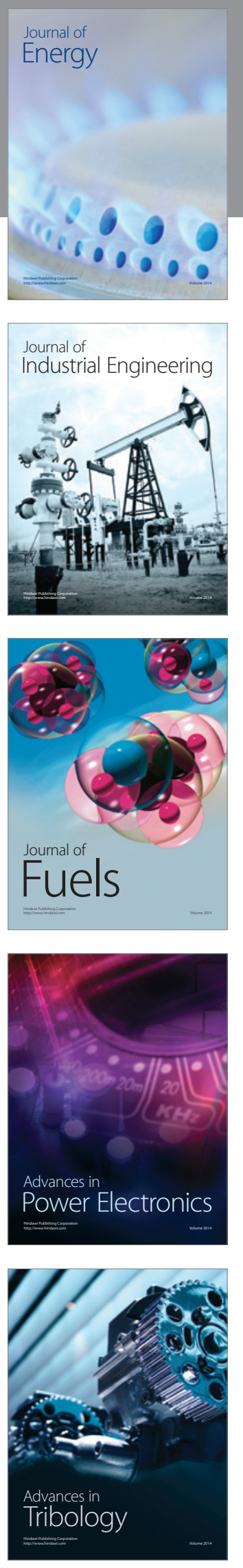
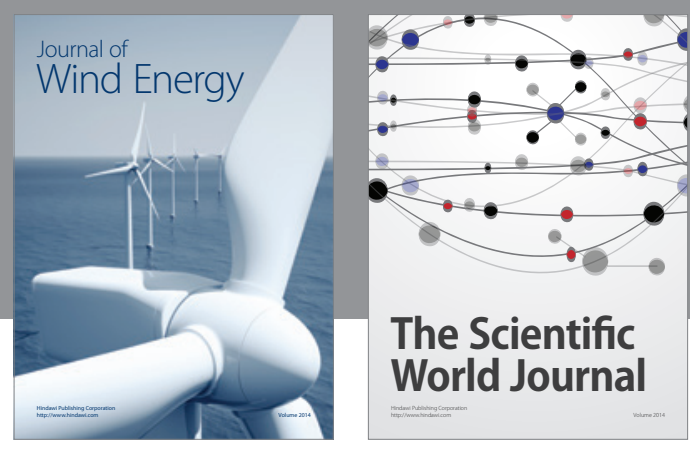

The Scientific World Journal

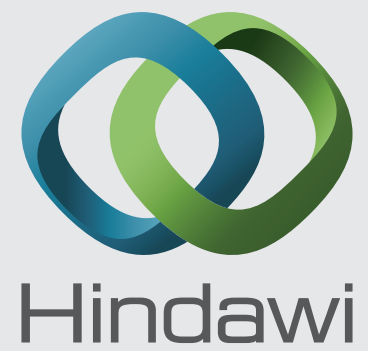

Submit your manuscripts at http://www.hindawi.com
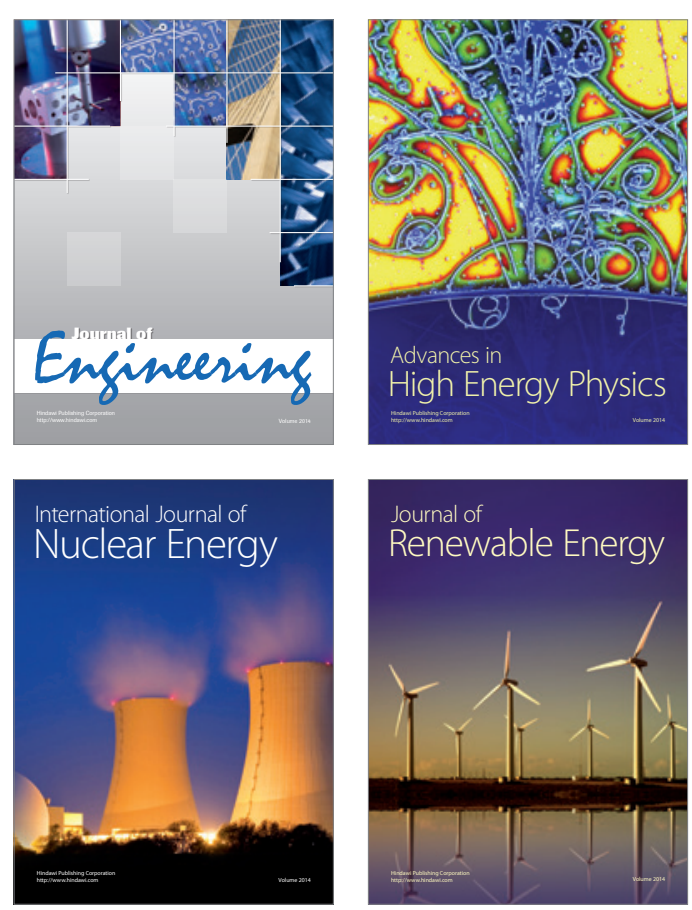

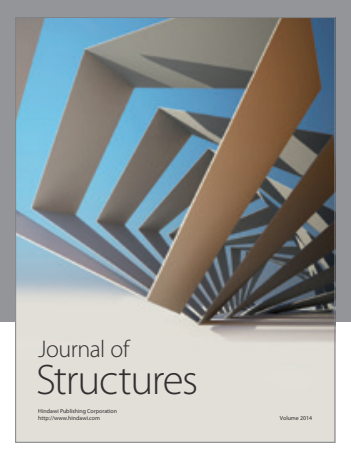

Rotating
Mechinery
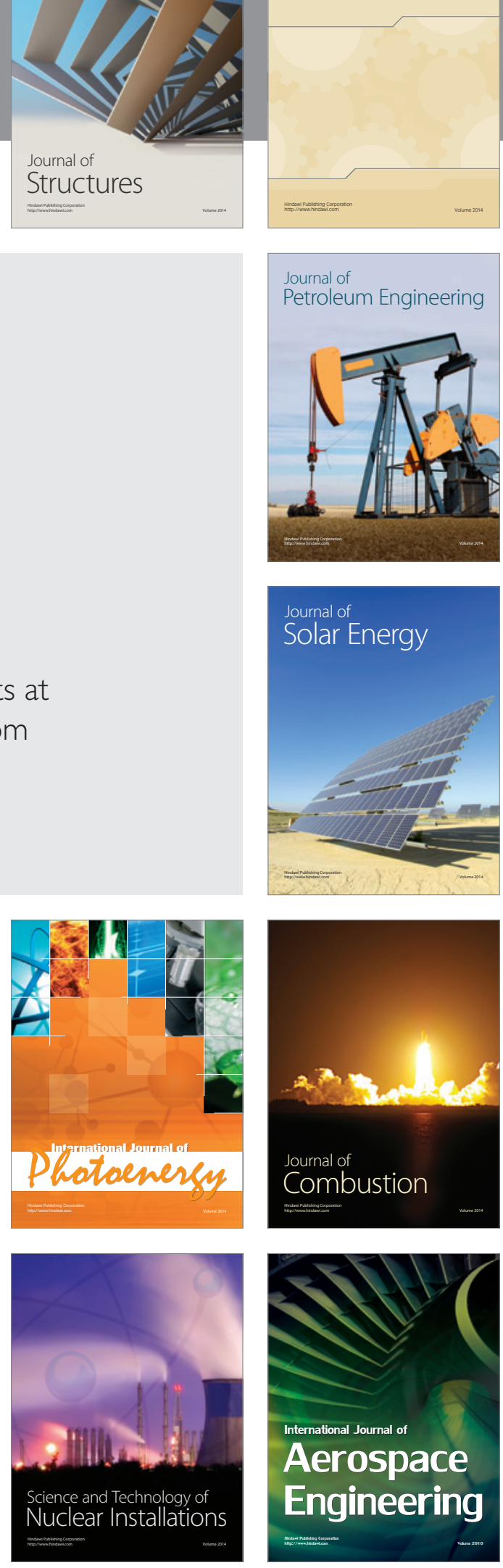